Copyright by the American Physical Society. Farkas, D.; Van Swygenhoven, H.; Derlet, P. M.,

"Intergranular fracture in nanocrystalline metals," Phys. Rev. B 66, 060101 (R) DOI: http://

PHYSICAL REVIEW B 66, 060101(R) (2002)

\title{
Intergranular fracture in nanocrystalline metals
}

\author{
D. Farkas, ${ }^{1}$ H. Van Swygenhoven, ${ }^{2}$ and P. M. Derlet ${ }^{2}$ \\ ${ }^{1}$ Department of Materials Science and Engineering, Virginia Polytechnic Institute and State University, Blacksburg, Virginia 24061-0237 \\ ${ }^{2}$ Paul Scherrer Institute, 5232 Villigen, Switzerland \\ (Received 31 May 2002; published 1 August 2002)
}

\begin{abstract}
Crack propagation studies in nanocrystalline Ni samples with mean grain sizes ranging from 5 to $12 \mathrm{~nm}$ are reported using atomistic simulations. For all grain sizes pure intergranular fracture is observed. Intergranular fracture is shown to proceed by the coalescence of microvoids formed at the grain boundaries ahead of the crack. The energy released during propagation is higher than the Griffith value, indicating an additional grain-boundary accommodation mechanism.
\end{abstract}

DOI: 10.1103/PhysRevB.66.060101

PACS number(s): 62.20.-x, 61.72.Mm, 62.25.+g

For many polycrystalline metallic materials, grain-size refinement represents a unique opportunity by which the material may be simultaneously strengthened and toughened. This is particularly attractive because most strengthening mechanisms result in an inverse relation between strength and fracture toughness. A crack propagating within one grain no longer satisfies the fracture criterion when it crosses to the neighboring grain. The crack then stops at the grain boundary (GB) and delays the propagation of fracture. As the grain size decreases, the crack will be stopped in this manner more effectively, explaining the beneficial role of structural refinement on fracture toughness. Thus for coarse-grain systems, the increase in fracture strength with decreasing grain size parallels a similar increase in yield strength with grain refinement, as given by the Hall-Petch relation, ${ }^{1,2}$ which is usually explained on the basis of dislocation pileup at the GB.

For nanocrystalline metals a breakdown of the Hall-Petch relation is expected at grain sizes such that a grain cannot support dislocation pileup. ${ }^{3-5}$ Moreover, for very small grain sizes, dislocation sources inside grains can hardly exist because of size and image force limitations. At these grain sizes a large fraction of atoms can be considered as GB atoms, therefore intergranular deformation mechanisms are expected to become relevant, as opposed to intragranular mechanisms based on dislocation activity. Computer simulations of plastic deformation in nanocrystalline fcc materials support this view. ${ }^{6-10}$ At the smallest grain sizes explored, all deformation is accommodated in the GB with a strain rate increasing for decreasing grain size under equal applied uniaxial tensile stress conditions. In this regime GB sliding is observed, the mechanism being triggered by atomic shuffling and to a lesser extent stress-assisted diffusion. ${ }^{10}$ In $\mathrm{Ni}$, for grain sizes above approximately $10 \mathrm{~nm}$, the strain rate becomes less dependent on grain size indicating a transition into a new regime involving intragrain partial dislocations that nucleate at the GB.

In this communication we explore the fracture behavior in nanocrystalline materials, investigating the possibility of a similar breakdown of the fracture toughness increase brought about by grain-size refinement. In particular, we show that at these small grain sizes the crack propagates via an intergranular mechanism as opposed to an intragranular dislocation based process. A perfectly brittle material is one that fails exactly satisfying Griffith criterion, where the energy released per unit length advance of the crack and unit length of the crack front is twice the surface energy. ${ }^{11}$ From an application point of view, however, materials with energy release rates several times higher than this are considered brittle. In the present results we find fracture toughness in this range, that are higher than the Griffith value, indicating an accommodation mechanism in the grain boundaries, while still characterizing an intergranular crack propagation mechanism and a brittle material from an applications point of view.

Atomistic computer simulations constitute an ideal tool for the study of fracture mechanisms in metallic materials ${ }^{12-16}$ since it provides a level of detail not accessible through experiment. In the present work, we apply computersimulation techniques to explore the fracture behavior of nanocrystalline $\mathrm{Ni}$ in the range of $5-12 \mathrm{~nm}$ grain sizes. The initial atomic configurations are generated using a Voronoi construction, as is described in detail in previous simulations. ${ }^{6,8}$ The grain boundaries present in these samples have been fully characterized in a previous work. ${ }^{9}$ For all types of misfit, a large degree of structural coherence is observed and misfit accommodation occurs in a regular pattern. The grain-boundary structures observed in these nanocrystalline samples are similar to what is expected in coarse-grain materials.

The isotropic elastic approximation was used to introduce a mode I semi-infinite crack in a nanocrystalline simulation block. For the purposes of simulations at the atomistic scale this represents crack lengths of macroscopic sizes. This solution is used to fix the positions of the outermost atoms away from the crack tip and the crack tip region is then relaxed and allowed to find the minimum-energy configuration via a standard conjugate gradient technique, whilst the boundary conditions (the continuum part) are updated accordingly. Periodic boundary conditions are used in the direction of the crack front. The initial crack is an atomically sharp wedge whose tip is located near the center of the simulation block. The applied stress intensity is varied to simulate the equilibrium configuration of the tip as the crack advances (a finite crack under constant load and growing would be subject to increasingly higher stress intensity factor as it grows).

The semi-infinite crack is incrementally loaded to higher stress intensities, starting from a value well below the Grif- 

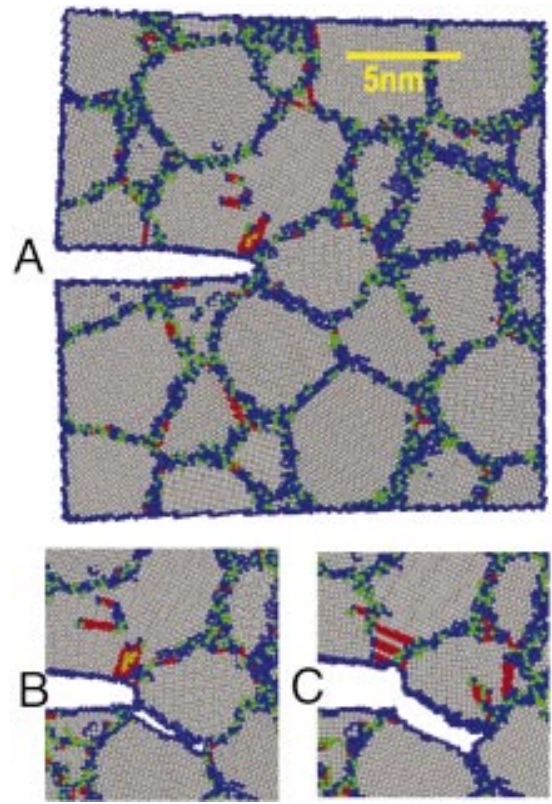

FIG. 1. (Color) Crack tip configuration in a section of a sample with $5 \mathrm{~nm}$ grain size. (A) At the Griffith load. (B) At a load of twice the Griffith value. (C) At a load of 2.5 times the Griffith value.

fith criterion, and the system is further relaxed for each value of applied loading. Such a molecular statics technique cannot address high temperature or dynamic effects of propagation at high values of applied strain rate, where the crack tip is not permitted to attain a stable configuration. Our simulations can be considered as snapshots of the quasiequilibrium crack tip region, as the macroscopic crack grows. This technique was similar to that used in previous studies of fracture behavior in single and bicrystals. ${ }^{15,16}$ In those studies, the focus was on the process of emission of dislocations from the crack tip and the interaction of the emitted dislocations with grain boundaries present in the bicrystals. In the present simulations we focus on the effects of the large amount of grain boundaries that are present in the nanocrystalline samples.

We carried out simulations for five different samples of average grain size from 5 to $12 \mathrm{~nm}$. These samples contain from $10^{5}$ to $4 \times 10^{6}$ atoms. The number of grains in the samples varied from 15 to 125 . The sizes of our simulations were chosen to be large enough to ensure that the fixed boundaries do not cause any spurious effects in the relaxation of the tip region.

We used an embedded atom method (EAM) potential for $\mathrm{Ni}$, based on first-principles calculations. ${ }^{17}$ This potential reproduces not only many equilibrium lattice and defect properties of fcc $\mathrm{Ni}$, but also the predictions of $a b$ initio calculations for various metastable phases for $\mathrm{Ni}$. The latter calculations span a wide range of configurations far from equilibrium and the transferability of this potential to situations very far from equilibrium is believed to be very good. The simulations were started with a stress intensity well below the Griffith critical condition. As the simulation progresses and the stress intensity is increased, the crack begins to advance, and we follow the crack for up to half the
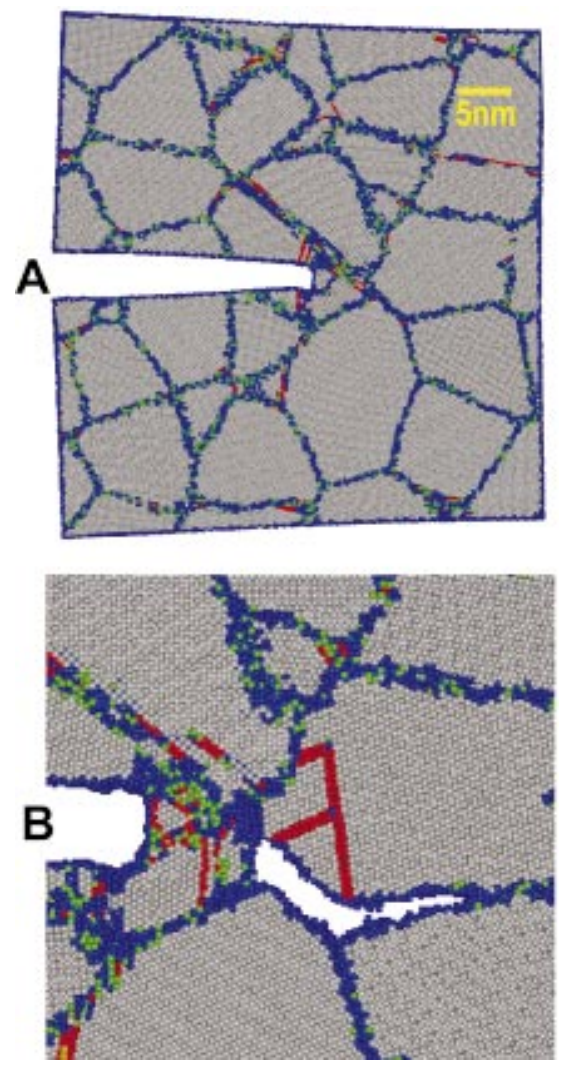

FIG. 2. (Color) Crack tip configuration in a section of a sample with $10 \mathrm{~nm}$ grain size. (A) At a load of twice the Griffith value. (B) At a load of 2.5 times the Griffith value.
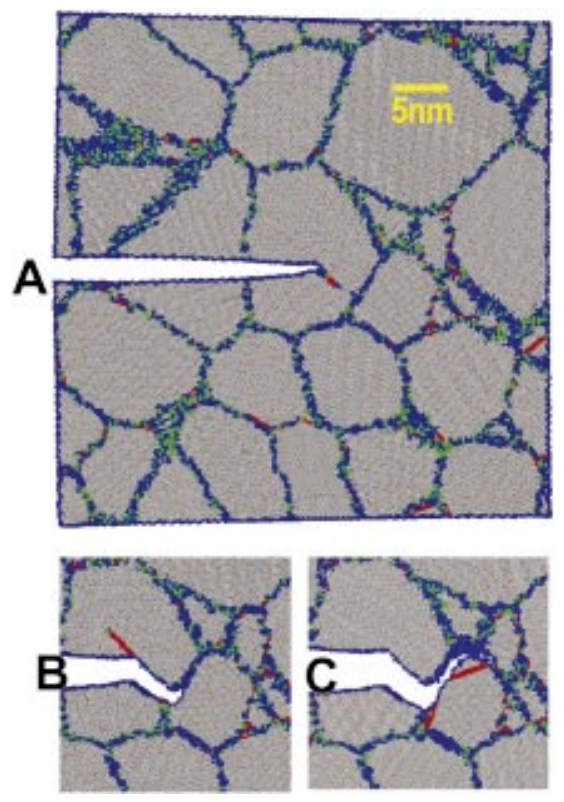

FIG. 3. (Color) Crack tip configuration in a different section of a sample with $10 \mathrm{~nm}$ grain size. (A) At the Griffith load. (B) At a load of twice the Griffith value. (C) At a load of 2.5 times the Griffith value. 


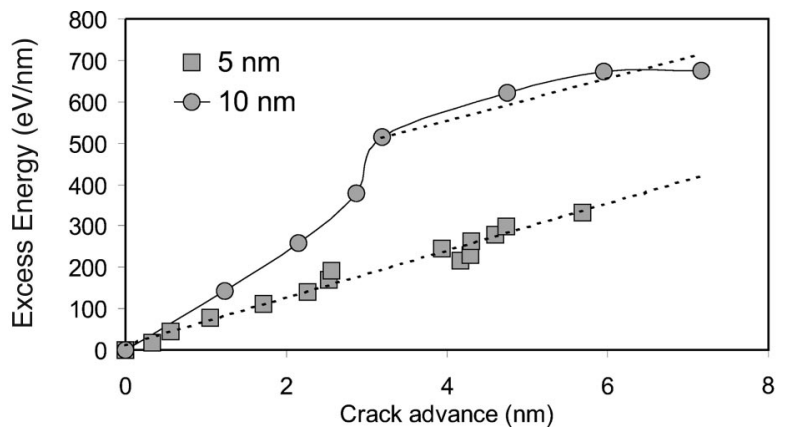

FIG. 4. Energy evolution as a function of crack growth for the 5-nm sample containing 125 grains and the $10 \mathrm{~nm}$, sample containing 60 grains. The slopes of the trend lines indicate the energy necessary for crack advance.

size of the sample in the direction of crack propagation. For visualization purposes, the local crystalline order of each atom is calculated using a short-range topological analysis, ${ }^{18}$ where in the following figures, gray represents fcc coordination, red represents hcp-type coordination, green represents other 12-coordinated local symmetries, and blue represent non-12-coordinated atoms. In this way the GB can be visualized via primarily the blue and green atoms. Local hcptype coordination of two subsequent $\{111\}$ planes occurs in stacking faults created by emitted Shockley partial dislocations and is therefore indicative of dislocation activity. For all figures displaying the entire sample, a layer of blue atoms is seen at the interface between the atomic and continuum region.

For the smaller grain-size samples $(5 \mathrm{~nm})$, the observed crack front, almost immediately, proceeds along paths entirely constituted by the grain boundaries of the sample. Figure 1 shows a cross section of the same sample configuration taken at different applied stresses along the crack front, namely, at 1 [Fig. 1(A)], 2 (Fig. 1(B)] and 2.5 [Fig. 1(C)] times the Griffith value. For this cross-sectional region, the tip of the initial wedge was in close proximity to a triple junction region. At the Griffith stress [Fig. 1(A)] the crack front remains at the triple junction region and blunting is observed. At two times the Griffith value [Fig. 1(B)], blunting is even more pronounced and the nucleation of two nanovoids ahead of the crack front can be seen. At larger loads [Fig. 1(C)], the crack then propagates along the intergranular path containing the nanovoids and stops at the next triple junction, where again blunting is observed. Only minor dislocation activity can be observed during the crack propagation, in the form of partial dislocations emitted from the grain-boundary or the triple junction region. The atomic scale mechanism, by which the crack both blunts and then continues to propagate, must therefore be primarily a grainboundary accommodation mechanism. In previous work, the atomic view of grain boundary sliding in nanocrystalline $\mathrm{Ni}$ at finite temperature using molecular dynamics, and under a constant tensile stress was adressed. ${ }^{10}$ In that work, two atomic processes were distinguished in the interfaces during sliding: atomic shuffling and stress-assisted free volume migration. The activated accommodation processes were found to be grain boundary and triple junction migration, and par- tial dislocation activity. In the present work, which employs molecular statics, these types of activated processes cannot be directly probed, but nevertheless similarities can be drawn from such a quasistatic approach.

For the larger grain size (10 and $12 \mathrm{~nm})$ studies, introduction of the wedge will introduce an atomically sharp crack tip that ends often in the inner regions of the grains. This somewhat artificial initial condition nevertheless gives further insight into the nature of crack propagation at the nanoscale regime. Figure 2 shows the atomic configurations of the crack in a $10 \mathrm{~nm}$ average grain-size sample, at the same cross-sectional position along the crack front, where Fig. 2(A) is for an applied load of 2 times the Griffith value, and Fig. 2(B) is a magnified region for an applied load of 2.5 times the Griffith value. For these grain sizes, the crack tip, initially within a grain, will emit a number of partial dislocations that blunt the tip region. Dislocation activity is, however, limited by the small grain size. Moreover nanovoids nucleate ahead of the crack front, in the neighboring grain boundaries to relieve local stress. At later stages these nanovoids join the main crack front. Where the crack tip is initially introduced in the vicinity of a grain boundary, the propagation proceeds along intergranular pathways. Intergranular crack propagation paths occur through the grain boundaries that are oriented favorably with respect the crack propagation direction. The grain boundaries that are close to perpendicular to the crack propagation direction actually arrest the crack. Partial dislocations, emitted from the grain boundary, can be observed in some grains situated along the crack path [Fig. 2(B)].

Figure 3 shows, for the same sample, another sequence of the crack propagation at an applied load twice the Griffith stress [Fig. 3(A)]. In this sequence, the crack is initially created with $\{100\}$ type faces that are not the lowest energy crystallographic orientation. The crack deflects to a more favorable $\{111\}$ plane within the grain and arrests at the nearby grain boundary. The mechanism by which it acheives this is via the emission, from the crack tip region, of several partial dislocations on a number of different slip planes. At 2.5 times the Griffith stress [Fig. 3(B)] the crack continues to propagate, now in an intergranular path.

By following the excess potential energy of the simulation block as a function of crack advancement we can introduce a simple method to obtain the overall average fracture toughness. Figure 4 shows two examples of the excess energy of the simulation block per unit length of crack front, plotted as a function of the crack average propagation distance. For the sample containing 125 grains and a 5-nm grain size the behavior is linear and the slope is approximately $59 \mathrm{eV} / \mathrm{nm}^{2}$ $\left(9.6 \mathrm{~J} / \mathrm{m}^{2}\right)$. For the larger grain size shown in the figure $(10$ $\mathrm{nm})$ the behavior is not linear, starting with a slope of $126 \mathrm{eV} / \mathrm{nm}^{2}\left(20.16 \mathrm{~J} / \mathrm{m}^{2}\right)$, during the initial propagation of the crack within the grains where the crack tip was initially located. However, as the crack reaches the nearest grain boundaries and begins to propagate intergranularly, the slope changes to $51 \mathrm{eV} / \mathrm{nm}^{2}$. This latter value is similar to that observed for the 5-nm sample shown in the figure $\left(59 \mathrm{eV} / \mathrm{nm}^{2}\right)$ and also compares well with the slope found for a different sample with the same grain size and 15 grains 
which was $48 \mathrm{eV} / \mathrm{nm}^{2}$. This analysis indicates that the process of intergranular fracture in nanocrystalline $\mathrm{Ni}$ has an associated energy release rate of around $50 \mathrm{eV} / \mathrm{nm}^{2}$ or $8 \mathrm{~J} / \mathrm{m}^{2}$. Assuming an average surface energy of $2.3 \mathrm{~J} / \mathrm{m}^{2}$, the observed energy release rate for the $5 \mathrm{~nm}$ grain-size samples is about twice higher than the expected bulk Griffith value of $4.6 \mathrm{~J} / \mathrm{m}^{2}$. Considering intergranular fracture and an average grain-boundary energy of $1.6 \mathrm{~J} / \mathrm{m}^{2}$ (Ref. 9) the Griffith value would be decreased by this amount, and equals to $3 \mathrm{~J} / \mathrm{m}^{2}$. The observed value represents then approximately three times the expected Griffith value for intergranular fracture. This is consistent with the fact that there is some dislocation activity in the adjacent grains even in the purely intergranular fracture case. Moreover, the GB accommodation mechanism that is responsible for the observed blunting at the GB and for the local changes on atomic level necessary for the stress relief, will also contribute to the additional energy release rate observed. In the larger grain sizes we do observe a transient regime of intragrain propagation. This has, however, to be ascribed to the artificial crack initiation. At these grain sizes, the initial crack ends inside the grain and has first to propagate to the GB. However, the grain boundaries located in the immediate vicinity of the crack tip begin to develop microcracks that lead to an intergranular fracture mechanism in these nanocrystalline materials. In the range of grain sizes studied, our results show a weak dependence of the mechanism on grain size. In conclusion, our results show that cracks propagate intergranularly in nanocrystalline Ni. When a crack tip is artificially made inside a grain, the crack initially blunts by a limited emission of partial dislocations, but soon this behavior is taken over by the creation of nanovoids in the surrounding grain boundaries, initiating an intergranular fracture mechanism, the nanovoids joining the main crack. In spite of the intergranular fracture mechanism observed, the fracture toughness observed is up to three times the expected Griffith value, indicating an energy release process. Since only very limited partial dislocation activity can be observed in grains along the crack, the results presented here give clear evidence of a grainboundary accommodation processes during fracture. Despite this energy release mechanism, the values of fracture tougnesses obtained indicate a brittle material from an applications point of view.

This work was supported by the office of Naval Research, Division of Materials Science and by the Swiss NSF (Grant No. 2000-056835.99)
${ }^{1}$ E.O. Hall, Proc. Phys. Soc. London, Sect. B 64, 747 (1951).

${ }^{2}$ N.J. Petch, J. Iron Steel Inst., London 174, 25 (1953).

${ }^{3}$ C.C. Koch, D.G. Morris, K. Lu, and A. Inoue, MRS Bull. 24, 54 (1999).

${ }^{4}$ J.R. Weertman, D. Farkas, K. Hemker, H. Kung, M. Mayo, R. Mitra, and H. Van Swygenhoven, MRS Bull. 24, 44 (1999).

${ }^{5}$ R.A. Masumura, P.M. Hazzledine, and C.S. Pande, Acta Mater. 46, 4527 (1998).

${ }^{6}$ H. Van Swygenhoven and A. Caro, Appl. Phys. Lett. 71, 1652 (1997).

${ }^{7}$ J. Schiotz, F.D. Di Tolla, and K.W. Jacobsen, Nature (London) 391, 561 (1998).

${ }^{8}$ H. Van Swygenhoven and A. Caro, Phys. Rev. B 58, 11246 (1998).

${ }^{9}$ H. Van Swygenhoven, D. Farkas, and A. Caro, Phys. Rev. B 62, 831 (2000).
${ }^{10}$ H. Van Swygenhoven and P.M. Derlet, Phys. Rev. B 64, 224105 (2001).

${ }^{11}$ A.A. Griffith, Philos. Trans. R. Soc. London, Ser. A 221, 163 (1921).

${ }^{12}$ D. Farkas, MRS Bull. 25, 38 (2000).

${ }^{13}$ S.J. Zhou, D.M. Beazley, and P.S. Lomdahl, and B.L. Holian, Phys. Rev. Lett. 78, 479 (1997).

${ }^{14}$ K. S Cheung and S. Yip, Phys. Rev. Lett. 65, 2804 (1990).

${ }^{15}$ R.K. Kalia, A. Nakano, A. Omeltchenko, K. Tsuruta, and P. Vashishta, Phys. Rev. Lett. 78, 2144 (1997).

${ }^{16}$ D. Farkas, Philos. Mag. Lett. 80, 229 (2000).

${ }^{17}$ Y. Mishin, D. Farkas, M.J. Mehl, and D.A. Papaconstantopoulos, Phys. Rev. B 59, 3393 (1999).

${ }^{18}$ D.J. Honeycutt and H.C. Andersen, J. Phys. Chem. 91, 4950 (1987). 